\title{
Nonlinear interactions in a cosmological background in the Dvali-Gabadadze-Porrati braneworld
}

\author{
Kazuya Koyama ${ }^{1}$ and Fabio P Silva ${ }^{1}$ \\ ${ }^{1}$ Institute of Cosmology and Gravitation, University of Portsmouth, Portsmouth PO1 2EG, United Kingdom \\ (Received 27 February 2007; published 27 April 2007)
}

\begin{abstract}
We study quasistatic perturbations in a cosmological background in the Dvali-Gabadadze-Porrati braneworld model. We identify the Vainshtein radius at which the nonlinear interactions of the brane bending mode become important in a cosmological background. The Vainshtein radius in the early universe is much smaller than the one in the Minkowski background, but in a self-accelerating universe it is the same as the Minkowski background. Our result shows that the perturbative approach is applicable beyond the Vainshtein radius for weak gravity by taking into account the second-order effects of the brane bending mode. The linearized cosmological perturbations are shown to be smoothly matched to the solutions inside the Vainshtein radius. We emphasize the importance of imposing a regularity condition in the bulk by solving the 5D perturbations and we highlight the problem of ad hoc assumptions on the bulk gravity that lead to different conclusions.
\end{abstract}

DOI: 10.1103/PhysRevD.75.084040

PACS numbers: $04.50 .+\mathrm{h}, 95.36 .+\mathrm{x}$

\section{INTRODUCTION}

The acceleration of the late-time universe is one of the most important problems in cosmology. Within the framework of general relativity, the acceleration is supposed to be caused by unknown dark energy. The simplest option for dark energy is vacuum energy, but it is hard to explain why the vacuum energy is so small compared with the prediction of particle physics. An alternative to dark energy is provided by models where large-distance modifications of gravity explain the acceleration. Probably the most widely studied example of a modified gravity model is the Dvali-Gabadadze-Porrati (DGP) braneworld model in which gravity leaks off the 4D brane into the 5D bulk spacetime [1]. The 5D action describing the DGP model is given by

$$
\begin{aligned}
S= & \frac{1}{2 \kappa^{2}} \int d^{5} x \sqrt{-} \bar{g}^{(5)} R+\frac{1}{2 \kappa_{4}^{2}} \int d^{4} x \sqrt{-\gamma} R \\
& +\int d^{4} x \sqrt{-\gamma} \mathcal{L}_{m}+\frac{1}{\kappa^{2}} \int d^{4} x \sqrt{-\gamma} K,
\end{aligned}
$$

where $\mathcal{L}_{m}$ is the Lagrangian for matter on the brane, $K_{\mu \nu}$ is the extrinsic curvature and $K=K_{\mu}^{\mu}$. The transition from $4 \mathrm{D}$ gravity to $5 \mathrm{D}$ gravity is governed by a crossover scale $r_{c}$,

$$
r_{c}=\frac{\kappa^{2}}{2 \kappa_{4}^{2}},
$$

which is the only parameter in this model. A striking feature of this model is the existence of a solution where the acceleration of universe is caused entirely by gravity without introducing the cosmological constant [2]. In this solution the Hubble parameter approaches a constant, $H \rightarrow$ $1 / r_{c}$, at late times, mimicking the cosmological constant. This self-accelerating solution has attracted significant interest recently [3].
Unfortunately, it has been shown that the selfaccelerating universe contains a ghost [4-9]. The existence of the ghost was shown rigorously on a de Sitter spacetime by studying linearized gravity. Recently, however, there are some claims that the nonlinear interactions obscure the conclusion on the existence of the ghost $[10,11]$. It has been recognized that the nonliner interactions of gravity in this model are much more subtle than 4D general relativity $[4,5,12-20]$. The reason is that the graviton contains a scalar degree of freedom and the nonlinear interaction of this mode becomes important on much larger scales than the usual graviton. This is analogous to the massive gravity model, where a helicity- 0 mode becomes strongly coupled on very large scales for small graviton mass [21]. In the DGP model, the scalar mode is a mix of the helicity-0 mode of the spin-2 5D graviton and the spin0 mode called the radion [6,7]. Physically, the scalar mode describes the bending of the brane in the bulk $[4,5,18]$. It was shown that the nonlinear interaction of the brane bending mode becomes important at the so-called Vainshtein radius $r_{*}=\left(r_{g} r_{c}^{2}\right)^{1 / 3}$, where $r_{g}$ is the Schwarzschild radius of the source [12]. If we want to explain the late-time acceleration, we should require $r_{c} \sim$ $H_{0}^{-1}$.

One argument against the validity of the linearized analysis is that, for cosmology, $r_{g}$ is roughly the Hubble scale today $r_{g} \sim H_{0}^{-1}$, then the Vainshtein radius is also the horizon scale $r_{*} \sim H_{0}^{-1}$, which may indicate that the linearized cosmological perturbations are not valid [10]. However, most of the literature so far studied perturbations around Minkowski spacetime. It is still unclear what is the Vainshtein radius in a cosmological background. This is an important question to be addressed because the ghost exists in the self-accelerating solution where the Minkowski spacetime is not a solution. There is no ghost in a Minkowski brane in the DGP model. Thus it is important 
to study nonlinear interactions of the brane bending mode in a Friedmann background.

There is also a claim that the perturbative approach cannot be applied in the DGP model [11]. This argument is based on the Schwarzschild solution obtained in Ref. [22], which does not recover the linearized solution in the region $r>r_{*}$. However, this solution is obtained by closing the equations on the brane by imposing ad hoc assumptions on the bulk gravity. In Ref. [23], it was shown that it is crucial to impose a proper boundary condition in the bulk to determine the behavior of gravity on the brane. The importance of imposing proper bulk boundary conditions was also emphasized in Ref. [24].

The aim of this paper is to study the nonlinear interactions of the brane bending mode on a Friedmann background. We build on Ref. [23] where linearized perturbations are solved properly in 5D spacetime. We extend the analysis of Ref. [23] by taking into account the second-order effects of the brane bending mode. Then we study whether the linearized cosmological perturbations can be smoothly matched to the solutions inside the Vainshtein radius. It should be noted that nonlinear interactions on a Friedmann background were studied in Ref. [16] assuming spherical symmetry and the modified Vainshtein radius was identified. We will confirm their result by properly solving the $5 \mathrm{D}$ metric perturbations without closing the equations on the brane in an heuristic way in the same spirit as Ref. [23]. For this purpose we closely follow the approach of Ref. [19], which studied weak gravity on the Minkowski background.

\section{QUASISTATIC PERTURBATIONS}

\section{A. Basic equations}

In this paper, we focus on weak gravity sourced by quasistatic matter fluctuations in a cosmological background. This analysis can be applied to describe the metric sufficiently far from a local source located in a cosmological background. We also can study the cosmological perturbations on subhorizon scales in the matter-dominated era, which are relevant for the structure formation problem.

The first-order metric in the bulk is given in a $5 \mathrm{D}$ longitudinal gauge by

$$
\begin{aligned}
d s^{2}= & -(1+2 \mathcal{A}) N(t, y)^{2} d t^{2} \\
& +(1+2 \mathcal{R}) A(t, y)^{2} \delta_{i j} d x^{i} d x^{j}+\left(1+2 A_{y y}\right) d y^{2},
\end{aligned}
$$

where

$$
A(y, t)=a(t)(1 \mp H y), \quad N(y, t)=1 \mp H\left(1+\frac{\dot{H}}{H^{2}}\right) y
$$

are the solutions for the background metric [2]. Note that the $(y, t)$ component of the metric can be neglected for a static source. The Hubble parameter is determined by the Friedmann equation and the continuity equation:

$$
\mp \frac{H}{r_{c}}=H^{2}-\frac{\kappa_{4}^{2}}{3} \rho, \quad \dot{\rho}+3 H(\rho+p) \rho=0 .
$$

The solution with - sign is called the normal branch solution and the solution with + sign is called the selfaccelerating solution because there is a de Sitter solution even without any kind of matter on the brane [2]. The selfaccelerating solution attracted significant interest as a model for dark energy from the large-distance modification of gravity.

In the 5D Longitudinal gauge, the brane is not located at $y=0$ [25]. Then it is more convenient to move to a gauge where the brane is located at $y=0$. We perform a gauge transformation $y \rightarrow y-r_{c} \xi$, where $\xi$ is a scalar function describing the perturbation of the brane location, which is often called the brane bending mode. The resultant metric has the form

$$
\begin{aligned}
d s^{2}= & -(1+2 \Psi) N(t, y)^{2} d t^{2} \\
& +(1+2 \Phi) A(t, y)^{2} \delta_{i j} d x^{i} d x^{j}+2 r_{c} \varphi_{, i} d x^{i} d y \\
& +(1+2 \Gamma) d y^{2} .
\end{aligned}
$$

At first order, $\varphi$ is identified as the brane bending mode $\xi$. We are interested in perturbations well inside the horizon. Thus we will neglect all subleading terms suppressed by $a H / k \ll 1$, where $k$ is the 3D wave number of the perturbations. Within quasistatic approximations, timederivative terms can be neglected. We also neglect terms like $\left(A^{\prime} / A\right) \Phi^{\prime}$ where prime denotes a derivative with respect to $y$. This is based on an assumption that $\Phi^{\prime} \sim k \Phi$. This assumption will be justified later. Although we are dealing with the linearized metric perturbations, it has been recognized that second-order terms of $\varphi$ can be important on larger scales compared with the other second-order contributions $[4,5,18,19]$. Thus we only keep the secondorder terms for $\varphi$. This assumption will also be verified later.

Under these assumptions, the 5D Einstein equations are given by

$$
\begin{aligned}
\delta^{(5)} G_{y}^{y}= & \frac{1}{A^{2}} \nabla^{2} \Psi+\frac{2}{A^{2}} \nabla^{2} \Phi-\frac{r_{c}}{A^{2}}\left(2 \frac{A^{\prime}}{A}+\frac{N^{\prime}}{N}\right) \nabla^{2} \varphi \\
& +\frac{r_{c}^{2}}{2 A^{4}}\left[\left(\nabla^{2} \varphi\right)^{2}-\left(\nabla_{i} \nabla_{j} \varphi\right)^{2}\right]=0,
\end{aligned}
$$

$$
\begin{aligned}
\delta^{(5)} G_{i}^{y}= & -\left(\Psi^{\prime}+2 \Phi^{\prime}\right)_{, i}-\frac{r_{c}^{2}}{2 A^{4}}\left[\left(\nabla^{j} \varphi\right)\left(\nabla_{j} \nabla_{i} \varphi^{\prime}\right)\right. \\
& \left.-\left(\nabla_{i} \varphi\right)\left(\nabla^{2} \varphi^{\prime}\right)\right]=0
\end{aligned}
$$

$$
\begin{aligned}
\delta^{(5)} G_{t}^{t}= & 3 \Phi^{\prime \prime}+\frac{2}{A^{2}} \nabla^{2} \Phi+\frac{\nabla^{2}}{A^{2}}\left(\Gamma-r_{c} \varphi^{\prime}\right)-2 \frac{r_{c}}{A^{2}}\left(\frac{A^{\prime}}{A}\right) \nabla^{2} \varphi \\
& +\frac{r_{c}^{2}}{2 A^{4}}\left[\left(\nabla^{2} \varphi\right)^{2}-\left(\nabla_{i} \nabla_{j} \varphi\right)^{2}\right]=0
\end{aligned}
$$




$$
\begin{aligned}
\delta^{(5)} G_{j}^{i}= & -\frac{1}{A^{2}}\left(\nabla^{i} \nabla_{j}-\delta_{j}^{i} \nabla^{2}\right)\left(\Phi+\Psi+\Gamma-r_{c} \varphi^{\prime}\right) \\
& +\delta_{j}^{i}\left(\Psi^{\prime \prime}+2 \Phi^{\prime \prime}\right)+\frac{r_{c}}{A^{2}}\left(\nabla^{i} \nabla_{j}-\delta_{j}^{i} \nabla^{2}\right)\left(\frac{A^{\prime}}{A}+\frac{N^{\prime}}{N}\right) \varphi \\
& -\frac{r_{c}^{2}}{A^{4}}\left[\left(\nabla^{2} \varphi\right)\left(\nabla^{i} \nabla_{j} \varphi\right)-\left(\nabla_{j} \nabla^{k} \varphi\right)\left(\nabla^{i} \nabla_{k} \varphi\right)\right] \\
& +\frac{1}{2} \delta_{j}^{i} \frac{r_{c}^{2}}{A^{4}}\left[\left(\nabla^{2} \varphi\right)^{2}-\left(\nabla_{k} \nabla_{l} \varphi\right)^{2}\right]=0 .
\end{aligned}
$$

For the spatial components $\delta^{(5)} G_{j}^{i}$, the trace of the equation gives

$$
\begin{aligned}
\frac{2}{A^{2}} \nabla^{2}\left(\Phi+\Psi+\Gamma-r_{c} \varphi^{\prime}\right)-\frac{2 r_{c}}{A^{2}}\left(\frac{A^{\prime}}{A}+\frac{N^{\prime}}{N}\right) \nabla^{2} \varphi \\
\quad+3\left(\Psi^{\prime \prime}+2 \Phi^{\prime \prime}\right)+\frac{1}{2} \frac{r_{c}^{2}}{A^{4}}\left[\left(\nabla^{2} \varphi\right)^{2}-\left(\nabla_{i} \nabla_{j} \varphi\right)^{2}\right]=0 .
\end{aligned}
$$

On the other hand, taking the divergence of the traceless part of $\delta^{(5)} G_{j}^{i}$, we get

$$
\begin{aligned}
\frac{\nabla^{2}}{A^{2}}(\Phi+ & \left.\Psi+\Gamma-r_{c} \varphi^{\prime}\right)-\frac{r_{c}}{A^{2}}\left(\frac{A^{\prime}}{A}+\frac{N^{\prime}}{N}\right) \nabla^{2} \varphi \\
+ & \frac{1}{4} \frac{r_{c}^{2}}{A^{4}}\left[\left(\nabla^{2} \varphi\right)^{2}-\left(\nabla_{i} \nabla_{j} \varphi\right)^{2}\right]=0 .
\end{aligned}
$$

The existence of the brane imposes the junction condition at the brane, that relates the extrinsic curvature with the energy-momentum tensor on the brane

$$
K_{\mu \nu}-K g_{\mu \nu}=-\frac{\kappa^{2}}{2} T_{\mu \nu}+r_{c} G_{\mu \nu} .
$$

We should note that due to the induced gravity term, the Einstein tensor appears in the junction condition. The $(t, t)$ component of the junction condition Eq. (2.11) gives

$$
\frac{2}{a^{2}} \nabla^{2} \Phi=-\kappa_{4}^{2} \delta \rho+\frac{1}{a^{2}} \nabla^{2} \varphi-\frac{3}{r_{c}} \Phi^{\prime} .
$$

The spatial components give

$$
\begin{gathered}
\Phi+\Psi=\varphi, \\
\Psi^{\prime}+2 \Phi^{\prime}=0 .
\end{gathered}
$$

\section{B. Solutions in the bulk}

Let us first solve the perturbations in the bulk. Combining Eq. (2.5) and (2.10), $\Phi$ and $\Gamma-r_{c} \varphi^{\prime}$ are written in terms of $\Psi$ and $\varphi$ :

$$
\begin{aligned}
\frac{\nabla^{2}}{A^{2}} \Phi= & -\frac{1}{2} \frac{\nabla^{2}}{A^{2}} \Psi+\frac{r_{c}}{2 A^{2}}\left(2 \frac{A^{\prime}}{A}+\frac{N^{\prime}}{N}\right) \nabla^{2} \varphi \\
& -\frac{r_{c}^{2}}{4 A^{4}}\left[\left(\nabla^{2} \varphi\right)^{2}-\left(\nabla_{i} \nabla_{j} \varphi\right)^{2}\right],
\end{aligned}
$$

$$
\frac{\nabla^{2}}{A^{2}}\left(\Gamma-r_{c} \varphi^{\prime}\right)=-\frac{1}{2} \frac{\nabla^{2}}{A^{2}} \Psi+\frac{r_{c}}{2 A^{2}}\left(\frac{N^{\prime}}{N}\right) \nabla^{2} \varphi .
$$

Consistency between Eqs. (2.6) and (2.15) requires

$$
\varphi^{\prime}=0, \quad \Psi^{\prime}+2 \Phi^{\prime}=0
$$

The latter is consistent with the junction condition Eq. (2.14). Then substituting Eqs. (2.15) and (2.16) into Eq. (2.7) and using Eq. (2.17), we get a wave equation for $\Psi$

$$
\Psi^{\prime \prime}+\frac{\nabla^{2}}{A^{2}} \Psi-\left(\frac{N^{\prime}}{N}\right) \frac{r_{c}}{A^{2}} \nabla^{2} \varphi=0 .
$$

By performing a Fourier transformation, the solution is given by

$$
\begin{aligned}
\mathcal{A} & =\Psi-\frac{N^{\prime}}{N} r_{c} \varphi \\
& =\left[c_{1}(1 \mp H y)^{ \pm k / a H}+c_{2}(1 \mp H y)^{\mp k / a H}\right],
\end{aligned}
$$

for a given $k$, with our approximation $k / a H \gg 1$. We impose the regularity condition in the bulk so that the perturbations do not diverge at $y \rightarrow \infty$ in the selfaccelerating branch, and $y=1 / H$ in the normal branch. This means that we take $c_{2}=0$ [23]. We should note that the regularity condition verifies our assumption that the terms like $\left(A^{\prime} / A\right) \Phi^{\prime}$ can be neglected compared with the terms like $\nabla^{2} \Phi / A^{2}$, with our approximation $k / a H \gg 1$.

\section{Equations on the brane}

Now we impose the junction conditions. From Eqs. (2.19) and (2.15), it is possible to show that

$$
\frac{\Phi^{\prime}}{r_{c}} \sim \frac{k}{a r_{c}} \Phi \ll \frac{k^{2}}{a^{2}} \Phi,
$$

for perturbations whose physical wavelengths are shorter than $r_{c}, k r_{c} / a \gg 1$. Thus we can neglect $\Phi^{\prime}$ in the junction condition Eq. (2.12). Then the projection of Eq. (2.5) on the brane and the junction conditions Eqs. (2.12) and (2.14) provide a closed set of equations on the brane for $\Phi, \Psi$, and $\varphi$. The effective Einstein equations are written as

$$
\begin{gathered}
\frac{2}{a^{2}} \nabla^{2} \Phi=-\kappa_{4}^{2} \delta \rho+\frac{1}{a^{2}} \nabla^{2} \varphi, \\
\Psi+\Phi=\varphi,
\end{gathered}
$$

and the equation of motion for $\varphi$ is given by

$$
3 \beta(t) \frac{\nabla^{2}}{a^{2}} \varphi+\frac{r_{c}^{2}}{a^{4}}\left[\left(\nabla^{2} \varphi\right)^{2}-\left(\nabla_{i} \nabla_{j} \varphi\right)^{2}\right]=\kappa_{4}^{2} \delta \rho,
$$

where

$$
\beta(t)=1-\frac{2 r_{c}}{3}\left(2 \frac{A^{\prime}}{A}+\frac{N^{\prime}}{N}\right)=1 \pm 2 H r_{c}\left(1+\frac{\dot{H}}{3 H^{2}}\right) .
$$


Here the + sign corresponds to the normal branch and the - sign to the self-accelerating one.

\section{SOLUTIONS ON A BRANE}

\section{A. Linearized solutions}

We begin with linearized solutions by neglecting the second-order contributions of $\varphi$. The solutions for the metric perturbations are easily obtained as

$$
\begin{gathered}
\frac{\nabla^{2}}{a^{2}} \Phi=-\frac{\kappa_{4}^{2}}{2}\left(1-\frac{1}{3 \beta}\right) \delta \rho, \\
\frac{\nabla^{2}}{a^{2}} \Psi=\frac{\kappa_{4}^{2}}{2}\left(1+\frac{1}{3 \beta}\right) \delta \rho,
\end{gathered}
$$

which agree with the solutions obtained in Refs. [16,23].

The linearized equations can be described by a BransDicke (BD) theory. The perturbed Einstein equations in the $\mathrm{BD}$ theory are given by

$$
\delta G_{\mu \nu}=-\left(\nabla_{\mu} \nabla_{\nu}-g_{\mu \nu} \nabla^{2}\right) \varphi,
$$

and the equation of motion for the BD scalar is

$$
\frac{\nabla^{2}}{a^{2}} \varphi=\frac{\kappa_{4}^{2}}{3+2 \omega} \delta \rho,
$$

where $\omega$ is the BD parameter. Comparing Eqs. (2.21), (2.22), and (2.23) with Eqs. (3.3) and (3.4), we find that the brane bending mode acts as the BD scalar and the BD parameter is given by $[16,23,26]$

$$
\omega=\frac{3}{2}(\beta-1) .
$$

The sign of $\beta$ is directly related to the existence of the ghost in de Sitter spacetime. In the self-accelerating branch, $\beta$ is negative for $H r_{c}>1 / 2$. In the BD theory, the BD scalar has the wrong sign for the kinetic term if $\omega<-3 / 2$, that is $\beta<0$. The condition that $\beta$ is negative is given by $H r_{c}>1 / 2$, which is precisely the condition for the existence of the ghost in de Sitter spacetime, as was shown in Refs. [4-6]. On the other hand, in the normal branch, $\beta$ is positive and we expect no ghost in this branch of the solutions.

\section{B. Spherically symmetric solutions}

Next, we study the effect of the second-order contributions of $\varphi$. Unfortunately, it is not easy to solve the equations for $\varphi$ with the nonlinear interactions. Thus we assume spherical symmetry to simplify the problem. The equation for $\varphi$ (2.23) is then given by

$$
\left(\frac{d^{2}}{d r^{2}}+\frac{2}{r} \frac{d}{d r}\right)(3 \beta \varphi+\Xi)=\kappa_{4}^{2} \delta \rho,
$$

where

$$
\Xi=2 r_{c}^{2} \int \frac{1}{r}\left(\frac{d \varphi}{d r}\right)^{2} d r,
$$

in agreement with Ref. [19] in a Minkowski spacetime. Let us consider a source localized in some compact region. Then it is possible to integrate the equation to get

$$
3 \beta \varphi+\Xi+\frac{r_{g}}{r}=0,
$$

where

$$
r_{g}=\kappa_{4}^{2} \int_{0}^{r} d r r^{2} \delta \rho
$$

is the Schwarzschild radius of the source. Hereafter, we assume $r_{g}=$ const, for simplicity. Taking the $r$ derivative of Eq. (3.8) gives an algebraic equation for $d \varphi / d r$. Then we get a solution for $d \varphi / d r$ as

$$
\frac{d \varphi}{d r}=\frac{r_{g}}{r^{2}} \Delta(r), \quad \Delta(r)=\frac{2}{3 \beta}\left(\frac{r}{r_{*}}\right)^{3}\left(\sqrt{1+\left(\frac{r_{*}}{r}\right)^{3}}-1\right),
$$

where

$$
r_{*}=\left(\frac{8 r_{c}^{2} r_{g}}{9 \beta^{2}}\right)^{1 / 3},
$$

which is the Vainshtein radius for a source in a cosmological background. This is in agreement with the result of Ref. [16], but we arrive at this result by solving the 5D bulk metric and imposing the regularity condition in the bulk, without closing the equations on the brane in an heuristic way. The solutions for the metric perturbations can be obtained as

$$
\begin{gathered}
\Phi=\frac{r_{g}}{2 r}+\frac{\varphi}{2}, \\
\Psi=-\frac{r_{g}}{2 r}+\frac{\varphi}{2} .
\end{gathered}
$$

On scales larger than the Vainshtein radius $r>r_{*}$, the solutions are given by

$$
\begin{gathered}
\Phi=\frac{r_{g}}{2 r}\left(1-\frac{1}{3 \beta}\right), \\
\Psi=-\frac{r_{g}}{2 r}\left(1+\frac{1}{3 \beta}\right) .
\end{gathered}
$$

which agree with the linearized solutions Eqs. (3.1) and (3.2). This shows that the linearized solutions do make sense as long as we are considering scales larger than the Vainshtein radius.

On scales smaller than the Vainshtein radius, $r<r_{*}$, the solutions for $\Psi$ and $\Phi$ are obtained as 


$$
\begin{gathered}
\Phi=\frac{r_{g}}{2 r}+\frac{1}{\beta} \sqrt{\frac{\beta^{2} r_{g} r}{2 r_{c}^{2}}}, \\
\Psi=-\frac{r_{g}}{2 r}+\frac{1}{\beta} \sqrt{\frac{\beta^{2} r_{g} r}{2 r_{c}^{2}}} .
\end{gathered}
$$

In this region, the corrections to the solution in $4 \mathrm{D}$ general relativity are suppressed for $r<r_{*}$ so that Einstein gravity is recovered. From Eq. (2.23), we can see that $\Xi$ dominates over the linear term in this region. This indicates that once $\varphi$ becomes nonlinear, the solutions for the metric approach those in $4 \mathrm{D}$ general relativity. We should note that $\beta$ is negative in the self-accelerating solution while $\beta$ is positive in the normal branch solution. Then the corrections to 4D general relativity solutions have opposite signs in these solutions, as was first pointed out in Ref. [15]. By a simple coordinate transformation, we can check that our solutions agree with the results of Ref. [16].

Even on scales smaller than the Vainshtein radius $r<r_{*}$, the induced metric perturbations are small as long as we consider scales larger than the Schwarzschild radius $r>$ $r_{g}$. This justifies our assumption of neglecting all secondorder contributions other than the second-order terms of $\varphi$. It should be emphasized also that the $(y, r)$ component of the metric, $r_{c} \varphi_{, r}$, is evaluated as

$$
\begin{aligned}
&\left(r_{c} \varphi_{, r}\right)^{2} \sim\left(\frac{r_{*}}{r}\right)^{3}\left(\frac{r_{g}}{r}\right), \text { for } r>r_{*}, \\
&\left(r_{c} \varphi_{, r}\right)^{2} \sim\left(\frac{r_{g}}{r}\right), \quad \text { for } r<r_{*} .
\end{aligned}
$$

The higher-order terms of $\varphi$ in the Einstein equations have higher-order powers of $r_{c} \varphi, r$. Thus they are suppressed for $r>r_{g}$. Then we only need to keep the second-order terms which can be comparable to the linear terms as is seen from Eqs. (3.17) and (3.19).

\section{Cosmological perturbations}

Finally, we consider cosmological perturbations in a matter-dominated universe. We define an overdensity of dark matter as

$$
\delta=\frac{\delta \rho}{\rho} .
$$

The continuity equation and the Euler equation are the same as in 4D general relativity:

$$
\begin{gathered}
\frac{\partial \delta}{\partial t}+\frac{1}{a} \nabla(1+\delta) \mathbf{v}=0, \\
\frac{\partial \mathbf{v}}{\partial t}+\frac{1}{a}(\mathbf{v} \cdot \nabla) \cdot \mathbf{v}+H \mathbf{v}=-\frac{1}{a} \nabla \Psi,
\end{gathered}
$$

where $\mathbf{v}$ is the velocity perturbation of dark matter. Here we introduce time-derivative terms. In order to ensure our quasistatic approximation, the time dependence of the overdensity $\delta$ should be weak, $\partial_{t} \delta \ll k \delta$, which is indeed valid for dust matter. Combining Eqs. (3.21) and (3.22) with Eqs. (2.21), (2.22), and (2.23), we can describe the evolution of the dark matter overdensity.

The nonlinear terms in the equation for $\varphi$, Eq. (2.23), become dominant when

$$
\frac{\beta a^{2}}{r_{c}^{2} k^{2}}<\varphi
$$

Using the linear term in Eq. (2.23), $\varphi$ is estimated as

$$
\varphi \sim \frac{H^{2} a^{2}}{\beta k^{2}} \delta,
$$

where we used $\kappa_{4}^{2} \rho \sim H^{2}$. Then the condition that the nonlinear terms become important is given in terms of $\delta$ by

$$
\beta^{2}\left(H r_{c}\right)^{-2} \sim O(1)<\delta .
$$

If nonlinear terms become dominant, $\varphi$ is estimated as

$$
\frac{k^{2}}{a^{2}} \varphi \sim\left(\frac{H^{2} a^{2}}{r_{c}^{2} k^{2}}\right) \delta .
$$

Then in the Poisson equation (2.21), the contribution of $\varphi$ can be neglected and 4D general relativity is recovered. Thus, from these rough estimations, we expect to recover 4D general relativity for nonlinear overdensity $\delta \gg 1$. This also means that for linear overdensity $\delta \ll 1$, the second-order terms of $\varphi$ can be neglected and the linearized cosmological perturbations do perfectly make sense as opposed to the claim made in Ref. [10]. In order to verify these estimations, one should solve the nonlinear equations for $\delta$ and $\varphi$, which is difficult even in conventional 4D general relativity. One approach is to consider the spherically symmetric collapse of the overdensity. This was done in Ref. [16], and it was demonstrated that once the overdensity exceeds $O(1)$, 4D general relativity is recovered. This confirms our estimations.

\section{EFFECTIVE THEORY ON THE BRANE}

\section{A. Effective theory for $\varphi$}

In the previous section, we find that the brane bending mode $\varphi$ plays a crucial role in the DGP model. It is possible to understand the role of the brane bending mode in a covariant way as was shown in Refs. [4,5]. We begin with the definition of the extrinsic curvature:

$$
K_{\mu \nu}=\frac{1}{2 \mathcal{N}}\left(\partial_{y} g_{\mu \nu}-\nabla_{\mu} N_{\nu}-\nabla_{\nu} N_{\mu}\right),
$$

where $g_{\mu \nu}$ is the induced metric, $N_{\mu}$ is a shift function, and $\mathcal{N}=\sqrt{g_{y y}-N_{\mu} N^{\mu}}$ is a lapse function. Let us first consider perturbations around Minkowski spacetime

$$
g_{\mu \nu}=\eta_{\mu \nu}+\delta g_{\mu \nu}
$$


The lapse function is given in terms of the brane bending mode $\varphi$ by $N_{\mu}=r_{c} \nabla_{\mu} \varphi$. Then the extrinsic curvature is given in terms of $\varphi$ by

$$
\delta K_{\mu \nu}=-r_{c} \nabla_{\mu} \nabla_{\nu} \varphi .
$$

An important result obtained by solving the 5D perturbations is that we can neglect the $y$ derivative of the induced metric in the junction condition because

$$
\partial_{y} g_{\mu \nu} \ll r_{c} \nabla^{2} \delta g_{\mu \nu}
$$

Then the junction condition becomes

$$
\delta G_{\mu \nu}=\kappa_{4}^{2} \delta T_{\mu \nu}-\left(\nabla_{\mu} \nabla_{\nu}-g_{\mu \nu} \nabla^{2}\right) \varphi .
$$

On the other hand, the Gauss equation in the bulk, that is the $(y, y)$ component of the 5D Einstein equations, gives

$$
R-K^{2}+K_{\mu \nu} K^{\mu \nu}=0 .
$$

Then combining Eqs. (4.5) and (4.6), we get the equation for $\varphi$ as

$$
3 \nabla^{2} \varphi+r_{c}^{2}\left[\left(\nabla^{2} \varphi\right)^{2}-\left(\nabla_{\mu} \nabla_{\nu} \varphi\right)^{2}\right]=-\kappa_{4}^{2} T,
$$

which reproduces Eq. (2.23) for static perturbations. We should emphasize that the nonlinear terms for $\varphi$ come from the nonlinear terms of $K_{\mu \nu}$. Even if we are dealing with weak gravity where the induced curvature is small, this does not necessarily mean that the nonlinearity of $K_{\mu \nu}$ can be neglected. We should also note that the higher-order terms in $\varphi$ comes from $N_{\mu} N^{\mu}$ which is given by $\left(r_{c} \varphi, r\right)^{2}$ in a spherically symmetric spacetime. We have shown that these contributions are suppressed as long as $r>r_{g}$.

In a cosmological background, the extrinsic curvature has contributions from the background

$$
K_{t t}=-\frac{N^{\prime}}{N} \quad K_{i j}=\frac{A^{\prime}}{A} \delta_{i j} .
$$

This gives an additional first-order contribution in the Gauss equation $[5,27]$

$$
\delta\left(-K^{2}+K_{\mu \nu} K^{\mu \nu}\right)=2 r_{c}\left(\frac{N^{\prime}}{N}+2 \frac{A^{\prime}}{A}\right) \nabla^{2} \varphi .
$$

This modifies the coefficient of the linear kinetic term for $\varphi$ to $3 \beta$. In de Sitter spacetime, this is exactly the origin of the ghost in the self-accelerating solution.

The equation of motion for $\varphi$ can be derived from the action

$$
S \propto-\int d^{4} x \sqrt{-\gamma}\left[3 \beta(\nabla \varphi)^{2}+r_{c}^{2}(\nabla \varphi)^{2} \nabla^{2} \varphi\right],
$$

assuming static perturbations. Defining a new field $\pi$ as $\pi=M_{4} \varphi$, where $\kappa_{4}^{2}=1 / M_{4}^{2}$, the action can be rewritten as

$$
S \propto-\int d^{4} x \sqrt{-\gamma}\left[3 \beta(\nabla \pi)^{2}+\frac{1}{\Lambda^{3}}(\nabla \pi)^{2} \nabla^{2} \pi\right],
$$

where $\Lambda=\left(M_{4} / r_{c}^{2}\right)^{1 / 3}$. In de Sitter spacetime, this agrees with the boundary effective action for the brane bending mode derived in Refs. [4,5]. Thus our solution is consistent with the effective theory for the brane bending mode of Refs. [4,5].

\section{B. Effective equation on the brane}

It is also possible to construct an effective theory for $\varphi$ using the effective equations on the brane. Projecting the 5D Einstein equations on the brane, the effective equations are given by [28]

$$
G_{\mu \nu}=\kappa^{4} \Pi_{\mu \nu}-E_{\mu \nu},
$$

where

$$
\begin{gathered}
\Pi_{\mu \nu}=-\frac{1}{4} \tilde{T}_{\mu \alpha} \tilde{T}_{\nu}^{\alpha}+\frac{1}{12} \tilde{T} \tilde{T}_{\mu \nu}+\frac{1}{8} g_{\mu \nu} \tilde{T}_{\alpha \beta} \tilde{T}^{\alpha \beta}-\frac{1}{24} g_{\mu \nu} \tilde{T}^{2}, \\
\tilde{T}_{\mu \nu}=T_{\mu \nu}-\kappa_{4}^{-2} G_{\mu \nu},
\end{gathered}
$$

and $E_{\mu \nu}$ is the projection of the electric part of the 5D Weyl tensor. For fluctuations around the vacuum Minkowski spacetime, $G_{\mu \nu}$ is written solely in terms of $\varphi$ from Eq. (4.5). Thus the effective equations are written in terms of $\varphi$ except for $E_{\mu \nu}$. The resultant effective equations are [29]

$$
\begin{aligned}
-\left(\nabla_{\mu} \nabla_{\nu}-g_{\mu \nu} \nabla^{2}\right) \varphi= & -\frac{r_{c}^{2}}{2}\left[g_{\mu \nu}\left\{\left(\nabla^{2} \varphi\right)^{2}-\left(\nabla_{\alpha} \nabla_{\beta} \varphi\right)^{2}\right\}\right. \\
& -2\left\{\left(\nabla^{2} \varphi\right)\left(\nabla_{\mu} \nabla_{\nu} \varphi\right)\right. \\
& \left.\left.-\left(\nabla_{\mu} \nabla_{\alpha} \varphi\right)\left(\nabla_{\nu} \nabla^{\alpha} \varphi\right)\right\}\right]-E_{\mu \nu}
\end{aligned}
$$

Taking the trace of this equation gives

$$
3 \nabla^{2} \varphi+r_{c}^{2}\left[\left(\nabla^{2} \varphi\right)^{2}-\left(\nabla_{\mu} \nabla_{\nu} \varphi\right)^{2}\right]=0,
$$

because $E_{\mu \nu}$ is traceless. This reproduces the equation of motion for $\varphi$, Eq. (2.23). On the other hand, the $(t, t)$ component gives

$$
3 \nabla^{2} \varphi+\frac{3}{2} r_{c}^{2}\left[\left(\nabla^{2} \varphi\right)^{2}-\left(\nabla_{\mu} \nabla_{\nu} \varphi\right)^{2}\right]=3 E_{t t} .
$$

If we neglected $E_{t t}$, this equation would contradict Eq. (4.16) as is pointed out by Ref. [29].

However, we should not neglect $E_{\mu \nu}$. From the 5D metric, $E_{t t}$ is calculated as

$$
E_{t t}=-3 \Phi^{\prime \prime}-\nabla^{2}\left(\Gamma-r_{c} \varphi^{\prime}\right) .
$$

It should be emphasized that $E_{t t}$ contains the second derivative of the metric with respect to $y$. Therefore, unless we solve the 5D perturbations, it is impossible to evaluate this term on the brane as the junction condition on the brane only determines the first derivatives. Using the solutions Eqs. (2.15) and (2.16) and the equations on the brane Eqs. (2.21), (2.22), and (2.23), $E_{t t}$ is evaluated as 


$$
E_{t t}=\frac{r_{c}^{2}}{6}\left[\left(\nabla^{2} \varphi\right)^{2}-\left(\nabla_{\mu} \nabla_{\nu} \varphi\right)^{2}\right] .
$$

Then it turns out that the $(t, t)$ component is fully consistent with Eq. (4.16). Thus the effective Eqs. (4.12) are consistent with our solutions. This is in fact trivial as the effective equations are nothing but the projection of 5D Einstein equations. Thus as long as we solve the 5D equations, the solutions should trivially satisfy the effective equations.

\section{Condition on $\boldsymbol{E}_{\boldsymbol{\mu \nu}}$}

At the linearized level, it was shown that the regularity condition for bulk perturbations gives a condition on $E_{\mu \nu}$ which cannot be determined by equations on the brane [23]. Here we check that this condition is not modified by the inclusion of nonlinear interactions of $\varphi$. First let us parameterize $E_{\mu \nu}$ as

$$
\delta E_{t}^{t}=\kappa_{4}^{2} \delta \rho_{E}, \quad \delta E_{j}^{i}=-\kappa_{4}^{2}\left[\frac{1}{3} \delta \rho_{E} \delta_{j}^{i}+\delta \pi_{E j}^{i}\right],
$$

where $\delta \pi_{E j}^{i}=\nabla^{i} \nabla_{j} \delta \pi_{E}-(1 / 3) \delta_{j}^{i} \nabla^{2} \pi_{E}$. In a cosmological background, these quantities are given by

$$
\begin{gathered}
\kappa_{4}^{2} \delta \rho_{E}=3 \Phi^{\prime \prime}+\frac{\nabla^{2}}{a^{2}}\left(\Gamma-r_{c} \varphi^{\prime}\right), \\
\kappa_{4}^{2} \delta \pi_{E}=\Gamma-r_{c} \varphi^{\prime} .
\end{gathered}
$$

Then using Eqs. (2.15), (2.16), and (2.18), it is possible to show that these satisfy the condition

$$
\delta \rho_{E}+2 \frac{\nabla^{2}}{a^{2}} \delta \pi_{E}=0 .
$$

Note that we have already used the regularity condition to assume $\Phi^{\prime} \sim k \Phi$ and neglect terms suppressed by $a H / k \ll 1$. This is exactly the condition obtained in Ref. [23]. As in the Minkowski case, we can evaluate $\delta \rho_{E}$ as

$$
\begin{aligned}
\kappa_{4}^{2} \delta \rho_{E}= & -\frac{1}{6} \frac{\left[1 \pm 2 H r_{c}\left(1+\frac{\dot{H}}{H^{2}}\right)\right]}{\left[1 \pm 2 H r_{c}\left(1+\frac{\dot{H}}{3 H^{2}}\right)\right]} \frac{r_{c}^{2}}{a^{4}}\left[\left(\nabla^{2} \varphi\right)^{2}\right. \\
& \left.-\left(\nabla_{i} \nabla_{j} \varphi\right)^{2}\right]+\frac{2}{3} \frac{\left[1 \pm 2 H r_{c}\left(1+\frac{\dot{H}}{2 H^{2}}\right)\right]}{\left[1 \pm 2 H r_{c}\left(1+\frac{\dot{H}}{3 H^{2}}\right)\right]} \kappa_{4}^{2} \delta \rho .
\end{aligned}
$$

This agrees with the result obtained in Ref. [23] if we neglect the second-order terms of $\varphi$. The $(t, t)$ component of the effective Einstein equation gives

$$
\begin{aligned}
\delta G_{t}^{t}= & 2 \frac{A^{\prime}}{A} \frac{r_{c}}{a^{2}} \nabla^{2} \varphi-\frac{r_{c}^{2}}{2 a^{4}}\left[\left(\nabla^{2} \varphi\right)^{2}-\left(\nabla_{i} \nabla_{j} \varphi\right)^{2}\right] \\
& -\kappa_{4}^{2} \delta \rho_{E},
\end{aligned}
$$

where we used the expressions for $\Pi_{\mu \nu}$ in terms of the extrinsic curvature

$$
\kappa^{4} \Pi_{\nu}^{\mu}=K K_{\nu}^{\mu}-K^{\mu \rho} K_{\nu \rho}-\frac{1}{2} \delta_{\nu}^{\mu}\left(K^{2}-K_{\alpha \beta} K^{\alpha \beta}\right),
$$

and Eqs. (4.3) and (4.8). Using the solution for $\delta \rho_{E}$, it is possible to check that this equation reduces to Eq. (2.21) using Eq. (2.22).

\section{CONCLUSION AND DISCUSSIONS}

In this paper, we studied quasistatic perturbations in a cosmological background in the DGP brane world. Using Gaussian coordinates, we derived the solutions for weak gravity by taking into account the nonlinear interactions of the brane bending mode. Solving the bulk metric perturbations and imposing a regularity condition, we got a closed set of equations, Eqs. (2.21), (2.22), and (2.23), on the brane. At the linearized level the theory is described by a $\mathrm{BD}$ theory with the BD parameter given by $\omega=3(\beta-$ 1) $/ 2$, where $\beta$ is given by Eq. (2.24). We studied the effects of nonliner interactions of the brane bending mode assuming spherical symmetry. We find that the Vainshtein radius at which nonlinear interactions of the bending mode become important is given by $r_{*}^{3}=r_{V}^{3} / \beta^{2}$ where $r_{V}$ is the Vainshtein radius in the Minkowski background. In the early universe, $\beta^{2} \gg 1$, so the Vainshtein radius is very small. Note that in this limit, we recover $4 \mathrm{D}$ general relativity even at the linearized level, as the BD parameter becomes large [26,27]. On the other hand, in the selfaccelerating universe, $H r_{c}=1, \quad \beta^{2}=1, \quad$ so the Vainshtein radius is the same as in the Minkowski background. On scales smaller than the Vainshtein radius, $r<$ $r_{*}$, the solution approaches 4D general relativity. Our solutions agree with the results of Refs. $[15,16]$ in the Friedmann background, and the results of Refs. $[14,18,19]$ in the Minkowski background.

Our equations can be applied to cosmological perturbations on subhorizon scales in the matter-dominated era. Although the nonlinear equations are difficult to solve in this case, we can estimate the scale at which the nonlinear interactions of the brane bending mode become important. We found that once the dark matter overdensity becomes nonlinear, the nonlinear terms of the bending mode also become important and the behavior of metric perturbations approach to $4 \mathrm{D}$ general relativity. This result is in accord with the finding in Ref. [16] where a spherical symmetric collapse is studied in the self-accelerating background. Our result indicates that the linearized cosmological perturbations analysis does make sense in the same way as in the conventional 4D cosmology as opposed to a claim made in Ref. [10].

We checked the consistency of our solutions with the effective equations on the brane. First, we checked that our solutions can be derived from the boundary effective theory for the bending mode derived in Refs. [4,5]. Following Ref. [29], we also checked the consistency of our solutions with the effective equations on the brane derived by a projection of 5D Einstein equations. A key quantity is 
the electric part of the bulk Weyl tensor projected onto the brane $E_{\mu \nu}$. If we neglected this Weyl contribution, the effective equations were inconsistent. Using the solutions in the bulk, we can evaluate $E_{\mu \nu}$ on the brane. We have shown that once the contribution from $E_{\mu \nu}$ is properly taken into account, the effective equations are fully consistent. Our analysis is consistent with the boundary effective action Eq. (4.11) at least for static perturbations. It was pointed out that this effective action manifests superluminal propagation if we consider time-dependent fluctuations around a spherically symmetric solution [30]. It would be important to extend our 5D analysis to include timedependent perturbations to check the validity of the boundary effective action with time-dependent perturbations and understand the causality of the propagation in the $5 \mathrm{D}$ spacetime.

Our conclusion is different from that of Ref. [11]. In Ref. [11], it is argued that the linearized perturbations, which by themselves are valid at $r>r_{*}$, are not guaranteed to match to the solution inside $r<r_{*}$. This argument is based on the Schwarzschild solution obtained in Ref. [22], which does not recover the linearized solution in the region $r>r_{*}$. However, the Schwarzschild solution in Ref. [22] is not derived by solving the bulk metric and imposing a proper boundary condition in the bulk. Instead they imposed a specific form of the metric and closed the equations on the brane. This is in fact the same as imposing an ad hoc condition on $E_{\mu \nu}$. As we have shown in this paper, the condition on $E_{\mu \nu}$ has to be determined by solving the bulk metric and imposing an appropriate boundary condition in the bulk. For weak gravity that is valid for $r>r_{g}$, the regularity condition in the bulk uniquely determines a condition for $E_{\mu \nu}$, Eq. (4.23). The Schwarzschild solution found in Ref. [22] does not satisfy this condition in the weak gravity region. Thus their solution is unlikely to describe weak gravity sourced by a physical local source on a brane. On the other hand, it is still an open question what is a proper condition on $E_{\mu \nu}$ for strong gravity. An outstanding open question is to find a fully nonlinear spherically symmetric solution that properly reproduces the solutions Eqs. (3.10) and (3.13) for weak gravity. We will come back to this issue in a separate paper.

Finally, we comment on the ghost problem. Our analysis shows that the linearized analysis does make sense as long as we consider scales beyond the Vainshtein radius $r_{*}$ for a local source. Then on scales $r>r_{*}$ we find a ghost in the self-accelerating universe. Usually, we expect an instant instability of the spacetime in the presence of the ghost. Then the self-accelerating universe would not be a viable background for cosmology. However, in this case, it is not so obvious that the ghost leads to an instant instability of the spacetime classically [31], or even quantum mechanically [32]. Furthermore, nonlinear interactions of the bending mode would become important if instabilities kick in. Further study is needed to understand the fate of this ghost. On the other hand, the normal branch solution is free from the ghost. Although the solution itself cannot be an alternative to dark energy, it still provides an interesting possibility to realize an expansion history of the Universe which is equivalent to a dark energy model with an equation of state less than -1 [33]. This model also provides a concrete example for the large-distance modification of gravity [8]. Our Eqs. (2.21), (2.22), and (2.23) are the basis for the study of structure formation tests in this model.

\section{ACKNOWLEDGMENTS}

K. K. is supported by PPARC. F.P.S. is supported by "Fundação para a Ciência e a Tecnologia (Portugal)," No. SFRH/BD/27249/2006. We would like to thank Roy Maartens for discussions and a careful reading of this manuscript.
[1] G. R. Dvali, G. Gabadadze, and M. Porrati, Phys. Lett. B 484, 112 (2000).

[2] C. Deffayet, Phys. Lett. B 502, 199 (2001).

[3] M. Ishak, A. Upadhye, and D. N. Spergel, Phys. Rev. D 74, 043513 (2006); M. Fairbairn and A. Goobar, Phys. Lett. B 642, 432 (2006); R. Maartens and E. Majerotto, Phys. Rev. D 74, 023004 (2006); S. Rydbeck, M. Fairbairn, and A. Goobar, astro-ph/0701495; Y. S. Song, I. Sawicki, and W. Hu, Phys. Rev. D 75, 064003 (2007).

[4] M. A. Luty, M. Porrati, and R. Rattazzi, J. High Energy Phys. 09 (2003) 029.

[5] A. Nicolis and R. Rattazzi, J. High Energy Phys. 06 (2004) 059.

[6] K. Koyama and K. Koyama, Phys. Rev. D 72, 043511 (2005); K. Koyama, Phys. Rev. D 72, 123511 (2005).
[7] D. Gorbunov, K. Koyama, and S. Sibiryakov, Phys. Rev. D 73, 044016 (2006).

[8] C. Charmousis, R. Gregory, N. Kaloper, and A. Padilla, J. High Energy Phys. 10 (2006) 066.

[9] K. Izumi, K. Koyama, and T. Tanaka, hep-th/0610282.

[10] G. Dvali, New J. Phys. 8, 326 (2006).

[11] C. Deffayet, G. Gabadadze, and A. Iglesias, J. Cosmol. Astropart. Phys. 08 (2006) 012.

[12] C. Deffayet, G.R. Dvali, G. Gabadadze, and A.I. Vainshtein, Phys. Rev. D 65, 044026 (2002).

[13] A. Lue, Phys. Rev. D 66, 043509 (2002).

[14] A. Gruzinov, New Astron. Rev. 10, 311 (2005).

[15] A. Lue and G. Starkman, Phys. Rev. D 67, 064002 (2003).

[16] A. Lue, R. Scoccimarro, and G. D. Starkman, Phys. Rev. D 69, 124015 (2004). 
[17] V. A. Rubakov, hep-th/0303125.

[18] T. Tanaka, Phys. Rev. D 69, 024001 (2004).

[19] C. Middleton and G. Siopsis, Mod. Phys. Lett. A 19, 2259 (2004).

[20] G. Dvali, G. Gabadadze, O. Pujolas, and R. Rahman, hepth/0612016.

[21] N. Arkani-Hamed, H. Georgi, and M.D. Schwartz, Ann. Phys. (N.Y.) 305, 96 (2003).

[22] G. Gabadadze and A. Iglesias, Phys. Rev. D 72, 084024 (2005).

[23] K. Koyama and R. Maartens, J. Cosmol. Astropart. Phys. 01 (2006) 016.

[24] A. Padilla, hep-th/0610093.

[25] C. Deffayet, Phys. Rev. D 66, 103504 (2002).

[26] K. Koyama and S. Mizuno, J. Cosmol. Astropart. Phys. 07 (2006) 013.

[27] C. Deffayet, Phys. Rev. D 71, 103501 (2005).
[28] T. Shiromizu, K.-i. Maeda, and M. Sasaki, Phys. Rev. D 62, 024012 (2000); K.-i. Maeda, S. Mizuno, and T. Torii, Phys. Rev. D 68, 024033 (2003); A. N. Aliev and A.E. Gumrukcuoglu, Classical Quantum Gravity 21, 5081 (2004).

[29] G. Gabadadze and A. Iglesias, Phys. Lett. B 639, 88 (2006).

[30] A. Adams, N. Arkani-Hamed, S. Dubovsky, A. Nicolis, and R. Rattazzi, J. High Energy Phys. 10 (2006) 014.

[31] I. Sawicki, Y.S. Song, and W. Hu, Phys. Rev. D 75, 064002 (2007).

[32] K. Izumi and T. Tanaka (private communication).

[33] V. Sahni and Y. Shtanov, J. Cosmol. Astropart. Phys. 11 (2003) 014; A. Lue and G. D. Starkman, Phys. Rev. D 70, 101501 (2004); R. Lazkoz, R. Maartens, and E. Majerotto, Phys. Rev. D 74, 083510 (2006). 